Published in J. Math. Phys., 38 (1997) p3399-3413

\title{
Novel generalization of three-dimensional Yang-Mills theory
}

\author{
Stephen C. Ancol \\ Department of Mathematics \\ University of British Columbia, Vancouver, BC Canada V6T $1 Z 1$ \\ and Department of Physics \\ University of Maryland, College Park, MD 20742
}

\begin{abstract}
A class of new nonabelian gauge theories for vector fields on three manifolds is presented. The theories describe a generalization of three-dimensional YangMills theory featuring a novel nonlinear gauge symmetry and field equations for Lie-algebra valued vector potential fields. The nonlinear form of the gauge symmetry and field equations relies on the vector cross-product and vector curl operator available only in three dimensions and makes use of an auxiliary Lie bracket together with the Lie bracket used in Yang-Mills theory. A gauge covariant formulation of the new theories is given which utilizes the covariant derivative and curvature from the geometrical formulation of Yang-Mills theory. Further features of the new theories are discussed.
\end{abstract}




\section{INTRODUCTION}

The wide ranging importance of gauge theories of vector fields in mathematics and physics raises interest in finding new types of these theories, especially generalizations of Yang-Mills theory. Recently, a new gauge theory [1] of this type has been constructed for vector fields on three-dimensional spacetimes. The theory can be understood to describe a nonlinear generalization of abelian Yang-Mills theory with the novel feature of a nonlinear gauge symmetry for abelian vector potential fields. The form of the gauge symmetry and field equations relies on vector cross-product and curl operations, limiting the generalization as a gauge theory of vector fields to work in three dimensions only.

This generalization is closely analogous in structure to the Freedman-Townsend [2] nonlinear generalization of abelian gauge theory for antisymmetric tensor fields on fourdimensional spacetimes. In particular, under the replacement of three-dimensional vector fields by d-dimensional antisymmetric tensor fields of rank d-2, the generalization of abelian Yang-Mills theory can be extended naturally as a gauge theory of antisymmetric tensor fields from three dimensions to d dimensions, where the four-dimensional theory is equivalent to the theory constructed by Freedman and Townsend. The structure of these theories as Yang-Mills theory generalizations suggests some extensions that can be pursued to give additional gauge theories of vector fields and antisymmetric tensor fields.

This paper is addressed to extending the Yang-Mills theory generalization in three dimensions from the abelian case to the nonabelian case, giving a class of new nonabelian gauge theories of vector fields on three-dimensional spacetimes, which was announced in Ref. [1]. The $S U(2)$ case of this generalization is worked out in Sec. II and results in a novel $S U(2)$ theory featuring a new form of nonlinear gauge symmetry and field equations. The construction of the theory relies on vector cross-product and curl operations available in three dimensions, and also uses the $S U(2)$ Lie algebra along with a related auxiliary Lie algebra. In contrast to the abelian case, where the analogous auxiliary Lie algebra is allowed to be arbitrary, the auxiliary Lie algebra in the $S U(2)$ case is fixed through an al- 
gebraic condition required by invariance of the action principle for the field equations under the gauge symmetry. As a consequence, in the $S U(2)$ case, the nonlinear structure of the generalization is unique.

Extension of the $S U(2)$ case to the general nonabelian case of the generalization is considered in Sec. III, which leads to a general class of nonabelian theories with features similar to the $S U(2)$ theory. In Sec. IV some additional features and extensions of the generalization are discussed.

\section{NEW SU(2) GAUGE THEORY}

\section{A. Formulation}

We start from the vector potential and field strength of $S U(2)$ Yang-Mills theory on a three-dimensional manifold. The structure we require on the manifold [3] is a metric $\eta_{\mu \nu}$ and a volume form $\epsilon_{\mu \nu \sigma}$ normalized with respect to metric, along with a compatible derivative operator $\partial_{\mu}$ determined by the metric. We also use the structure of the Lie algebra of $S U(2)$ in an arbitrary fixed basis $\left\{\mathbf{e}_{a}\right\}_{a=1,2,3}$, with Lie algebra multiplication $\left[\mathbf{e}_{a}, \mathbf{e}_{b}\right]=\epsilon_{a b}{ }^{c} \mathbf{e}_{c}$ and Killing metric $\left(\mathbf{e}_{a}, \mathbf{e}_{b}\right)=\delta_{a b}$. We fix the Killing metric components $\delta_{a b}$ in terms of the multiplication structure constants $\epsilon_{a b}{ }^{c}$ by $\epsilon_{a d}{ }^{e} \epsilon_{b e}{ }^{d}=-2 \delta_{a b}$, and we denote the components of the inverse of the Killing metric by $\delta^{a b}$, with $\delta_{a b} \delta^{b c}=\delta_{a}{ }^{c}$ denoting the components of the identity operator in the $S U(2)$ Lie algebra. In addition, we denote the inverse of $\eta_{\mu \nu}$ by $\eta^{\mu \nu}$, where $\eta_{\mu \nu} \eta^{\nu \sigma}=\delta_{\mu}{ }^{\sigma}$ is the identity tensor on the manifold. (Throughout, latin letters are used for internal Lie algebra indices, and greek letters are used for manifold coordinate indices, with all indices running from 1 to 3.)

We represent the vector potential in the $S U(2)$ basis by a set of cotangent vector fields $\left\{A_{\mu}^{a}\right\}_{a=1,2,3}$ and similarly represent the field strength of the vector potential by a set of antisymmetric tensor fields $\left\{F_{\mu \nu}^{a}\right\}_{a=1,2,3}$. The field strength tensors $F_{\mu \nu}^{a}$ are given in terms of the fields $A_{\mu}^{a}$ by 


$$
F_{\mu \nu}^{a}=\partial_{[\mu} A_{\nu]}^{a}+\frac{1}{2} \epsilon_{b c}^{a} A_{\mu}^{b} A_{\nu}^{c}
$$

The dual of these tensors are vectors $F_{\sigma}^{a}=\epsilon_{\sigma}{ }^{\mu \nu} F_{\mu \nu}^{a}$ which are constructable entirely by curl and cross-product operations along with $S U(2)$ multiplication,

$$
F_{\sigma}^{a}=\epsilon_{\sigma}{ }^{\mu \nu} \partial_{\mu} A_{\nu}^{a}+\frac{1}{2} \epsilon_{b c}{ }^{a} \epsilon_{\sigma}{ }^{\mu \nu} A_{\mu}^{b} A_{\nu}^{c}
$$

where $\epsilon_{\sigma}{ }^{\mu \nu}=\epsilon_{\sigma \alpha \beta} \eta^{\mu \alpha} \eta^{\nu \beta}$ is the cross-product tensor and $\epsilon_{\sigma}{ }^{\mu \nu} \partial_{\mu}$ is the curl operator.

To proceed with the construction of the new $S U(2)$ gauge theory, we introduce structure constants $\mathrm{B}_{a b}{ }^{c}$ defining an additional Lie algebra multiplication of the $S U(2)$ basis vectors $\mathbf{e}_{a}$. This multiplication will later be fixed through an algebraic condition imposed by gauge invariance. Using the structure constants, we now construct the field tensors

$$
Y_{\tau b}^{d \nu}=\delta_{\tau}{ }^{\nu} \delta_{b}{ }^{d}-\epsilon_{\tau}{ }^{\nu \sigma} \mathrm{B}^{d}{ }_{b e} A_{\sigma}^{e}
$$

linearly in terms of $A_{\sigma}^{e}$, where

$$
\mathrm{B}^{d}{ }_{b e}=\delta^{d a} \delta_{c e} \mathrm{~B}_{a b}^{c}
$$

The tensors $Y_{\tau b}^{d \nu}$ represent components of a linear map $Y$ on Lie-algebra valued cotangent vectors. We define tensors $Y_{\mu d}^{-1 a \tau}$ to represent components of the inverse linear map $Y^{-1}$, such that

$$
Y_{\mu d}^{-1 a \tau} Y_{\tau b}^{d \nu}=\delta_{b}{ }^{a} \delta_{\mu}^{\nu}=Y_{\mu d}^{a \tau} Y_{\tau b}^{-1 d \nu}
$$

with $A_{\sigma}^{e}$ restricted by the condition $\operatorname{det}(Y) \neq 0$ necessary for invertibility of $Y$. We also define the associated tensors

$$
\begin{aligned}
& Y_{a b}^{\mu \nu}=\delta_{a d} \eta^{\mu \tau} Y_{\tau b}^{d \nu}=\delta_{a b} \eta^{\mu \nu}-\epsilon_{\sigma}{ }^{\mu \nu} \mathrm{B}_{a b}{ }^{c} \delta_{c e} A_{\sigma}^{e} \\
& Y_{c d}^{-1 \sigma \tau}=\delta_{c a} \eta^{\sigma \mu} Y_{\mu d}^{-1 a \tau}
\end{aligned}
$$

which represent components of nondegenerate bilinear forms on Lie-algebra valued cotangent vectors. The bilinear forms are symmetric $Y_{a b}^{\mu \nu}=Y_{b a}^{\nu \mu}$ and $Y_{c d}^{-1 \sigma \tau}=Y_{d c}^{-1 \tau \sigma}$ due to the antisymmetry $\epsilon_{\sigma}{ }^{\mu \nu}=\epsilon_{\sigma}{ }^{[\mu \nu]}$ and $\mathrm{B}_{a b}{ }^{c}=\mathrm{B}_{[a b]}{ }^{c}$. 
From the previous structure the theory is constructed as follows. Field strength vectors in the theory are defined by

$$
K_{\mu}^{a}=Y_{\mu b}^{-1 a \nu} F_{\nu}^{b}
$$

In terms of these field strengths the Lagrangian of the theory is given quadratically by

$$
L=Y_{c d}^{-1 \sigma \tau} F_{\sigma}^{c} F_{\tau}^{d}=Y_{a b}^{\mu \nu} K_{\mu}^{a} K_{\nu}^{b}
$$

The gauge symmetry of the theory is given by the infinitesimal transformations

$$
\delta A_{\mu}^{a}=\partial_{\mu} \xi^{a}+\left(\epsilon_{b c}{ }^{a} A_{\mu}^{b}+\mathrm{B}_{d c}^{a} K_{\mu}^{d}\right) \xi^{c}
$$

involving a set of arbitrary functions $\xi^{a}$.

Gauge invariance of the theory requires $L$ to vary into a complete divergence $\partial_{\nu} S^{\nu}$ under an arbitrary gauge transformation $\delta A_{\mu}^{a}$, where $S^{\nu}$ is some local function of $A_{\mu}^{a}$, $\xi^{a}$, and their derivatives. This requirement now leads to the algebraic condition fixing the structure constants $\mathrm{B}_{a b}{ }^{c}$.

Consider an arbitrary infinitesimal variation of $L$,

$$
\delta L=\delta\left(Y_{a b}^{-1 \mu \nu} F_{\mu}^{a} F_{\nu}^{b}\right)
$$

Varying $F_{\mu}^{a}$ contributes the terms

$$
\begin{aligned}
2 Y_{a b}^{-1 \mu \nu} \delta F_{\mu}^{a} F_{\nu}^{b} & =2 \eta^{\sigma \mu} \delta_{c a} K_{\sigma}^{c} \delta F_{\mu}^{a} \\
& =2 K_{\sigma}^{c} \epsilon^{\sigma \nu \tau} \delta_{c a}\left(\partial_{\nu} \delta A_{\tau}^{a}+\epsilon_{d e}{ }^{a} A_{\nu}^{d} \delta A_{\tau}^{e}\right)
\end{aligned}
$$

and varying $Y_{a b}^{-1 \mu \nu}$ contributes the terms

$$
\begin{aligned}
\delta Y_{a b}^{-1 \mu \nu} F_{\mu}^{a} F_{\nu}^{b} & =-F_{\mu}^{a} F_{\nu}^{b} Y_{\sigma a}^{-1 c \mu} Y_{\tau b}^{-1 d \nu} \delta Y_{c d}^{\sigma \tau} \\
& =K_{\sigma}^{c} K_{\tau}^{d}\left(\epsilon^{\sigma \tau \nu} \mathrm{B}_{c d e} \delta A_{\nu}^{e}\right)
\end{aligned}
$$

through use of Eqs. 2.5) to (2.7), with $\epsilon^{\sigma \tau \nu}=\eta^{\sigma \mu} \epsilon_{\mu}{ }^{\tau \nu}$ and $\mathrm{B}_{c d e}=\mathrm{B}_{c d}{ }^{b} \delta_{b e}$. We now substitute the gauge symmetry (2.10) for the variations $\delta A_{a}^{\mu}$ and group the terms into a quadratic expression in powers of $\mathrm{B}_{a b}{ }^{c}$ and $\epsilon_{a b}{ }^{c}$. 
The linear part involving $\mathrm{B}_{a b}^{c}$ in $\delta L$ consists of the terms

$$
\begin{aligned}
& 2 \epsilon^{\nu \sigma \tau} \mathrm{B}_{b d e} K_{\nu}^{b} \partial_{\sigma}\left(K_{\tau}^{d} \xi^{e}\right)+\epsilon^{\nu \tau \sigma} \mathrm{B}_{b d e} K_{\nu}^{b} K_{\tau}^{d} \partial_{\sigma} \xi^{e} \\
& =\epsilon^{\nu \sigma \tau} \mathrm{B}_{b d e} \partial_{\sigma}\left(\xi^{e} K_{\nu}^{b} K_{\tau}^{d}\right)
\end{aligned}
$$

which combine to yield a complete divergence, using the antisymmetry property of Lie algebra multiplication, $\mathrm{B}_{b d}{ }^{a}=\mathrm{B}_{[b d]}{ }^{a}$. The quadratic part involving $\mathrm{B}_{a b}{ }^{c}$ in $\delta L$ is given by one term

$$
\mathrm{B}_{b c}^{e} \mathrm{~B}_{e d n} \xi^{n} \epsilon^{\nu \sigma \tau} K_{\nu}^{b} K_{\sigma}^{c} K_{\tau}^{d}
$$

The antisymmetry $\epsilon^{\nu \sigma \tau}=\epsilon^{[\nu \sigma \tau]}$ implies the product $\mathrm{B}_{b c}{ }^{e} \mathrm{~B}_{e d n}$ is antisymmetric in its indices $b c d$ and thus vanishes by the Jacobi property of Lie algebra multiplication, $\mathrm{B}_{[b c}{ }^{e} \mathrm{~B}_{|e| d]}{ }^{a}=0$. Consequently, the term (2.15) vanishes. Next, turning to the terms from the linear and quadratic parts involving $\epsilon_{a b}^{c}$ in $\delta L$ yields

$$
\begin{aligned}
& 2 \epsilon^{\nu \sigma \tau} K_{\nu}^{c}\left(\epsilon_{d e c} \partial_{\sigma}\left(A_{\tau}^{d} \xi^{e}\right)+\epsilon_{d e c} A_{\tau}^{e} \partial_{\sigma} \xi^{d}+\epsilon_{d e c} \epsilon_{m n}{ }^{d} A_{\tau}^{e} A_{\sigma}^{m} \xi^{n}\right) \\
& =2 \epsilon^{\nu \sigma \tau} K_{\nu}^{c}\left(\epsilon_{d e c} \partial_{\sigma} A_{\tau}^{d} \xi^{e}+\frac{1}{2} \epsilon_{d n c} \epsilon_{e m}{ }^{d} A_{\tau}^{e} A_{\sigma}^{m} \xi^{n}\right) \\
& =2 \epsilon_{d e c} \eta^{\nu \mu} K_{\nu}^{c} F_{\mu}^{d} \xi^{e}
\end{aligned}
$$

after we have rearranged $2 \epsilon_{d[e|c|} \epsilon_{m] n}{ }^{d}=\epsilon_{d n c} \epsilon_{e m}{ }^{d}$ by the Jacobi property of $S U(2)$ multiplication, where $\epsilon_{d e c}=\epsilon_{d e}^{b} \delta_{b c}$. Using the relations (2.3) and (2.8), we express

$$
F_{\mu}^{d}=Y_{\mu a}^{d \sigma} K_{\sigma}^{a}=K_{\mu}^{d}-\epsilon_{\mu}{ }^{\sigma \tau} \mathrm{B}^{d}{ }_{a m} K_{\sigma}^{a} A_{\tau}^{m}
$$

in the term (2.16), which leads to

$$
2 \epsilon_{d e c} \eta^{\nu \mu} K_{\nu}^{c} F_{\mu}^{d} \xi^{e}=2 \epsilon_{c d e} \eta^{\nu \mu} K_{\nu}^{c} K_{\mu}^{d} \xi^{e}-2 \epsilon_{e c}{ }^{d} \mathrm{~B}_{d a m} \epsilon^{\nu \sigma \tau} K_{\nu}^{c} K_{\sigma}^{a} A_{\tau}^{m} \xi^{e}
$$

by the $S U(2)$ multiplication relation $\epsilon_{d e c}=\epsilon_{c d e}=\epsilon_{e c d}$. The antisymmetry $\epsilon_{c d e}=\epsilon_{[c d] e}$ implies the term involving $2 \epsilon_{c d e} \eta^{\nu \mu} K_{\nu}^{c} K_{\mu}^{d}$ in Eq. (2.18) vanishes. Finally, the other term from Eq. (2.18) can be combined with all the terms in $\delta L$ from the remaining quadratic parts, 


$$
\begin{aligned}
& -2 \epsilon^{\nu \sigma \tau} \mathrm{B}_{d a m} \epsilon_{e c}{ }^{d} K_{\nu}^{c} K_{\sigma}^{a} A_{\tau}^{m} \xi^{e}+2 \epsilon^{\nu \sigma \tau} \mathrm{B}_{d m n} \epsilon_{e b}{ }^{d} K_{\nu}^{b} K_{\sigma}^{m} A_{\tau}^{e} \xi^{n}+\epsilon^{\nu \sigma \tau} \mathrm{B}_{b c e} \epsilon_{m n}{ }^{e} K_{\nu}^{b} K_{\sigma}^{c} A_{\tau}^{m} \xi^{n} \\
& =\epsilon^{\nu \sigma \tau} K_{\nu}^{b} K_{\sigma}^{c} A_{\tau}^{a} \xi^{d} H_{b c a d}
\end{aligned}
$$

where we have defined

$$
H_{b c a d}=2 \epsilon_{a[b}^{e} \mathrm{~B}_{|e| c] d}-2 \epsilon_{d[b}^{e} \mathrm{~B}_{|e| c] a}+\epsilon_{a d}^{e} \mathrm{~B}_{b c e}
$$

Assembling the previous terms in the gauge symmetry variation $\delta L$ now yields

$$
\delta L=\partial_{\sigma}\left(\epsilon^{\sigma \tau \nu} \mathrm{B}_{b d e} K_{\nu}^{b} K_{\tau}^{d} \xi^{e}\right)+\epsilon^{\nu \sigma \tau} K_{\nu}^{b} K_{\sigma}^{c} A_{\tau}^{a} \xi^{d} H_{b c a d}
$$

which is required to equal a complete divergence. Because the second term involves no derivatives of $\xi^{d}$, it is not a divergence and therefore must vanish. This implies

$$
0=H_{b c a d}
$$

constituting an algebraic condition on the structure constants $\mathrm{B}_{a b}{ }^{c}$.

We now solve Eq. (2.22) to determine $\mathrm{B}_{a b}{ }^{c}$ subject to the structure constant properties

$$
\mathrm{B}_{a b}^{c}=\mathrm{B}_{[a b]}^{c}, \mathrm{~B}_{[a b}^{d} \mathrm{~B}_{c] d}^{e}=0
$$

We contract Eq. (2.22) with $\epsilon_{n}{ }^{a d}$ and use the $S U(2)$ identities

$$
\begin{aligned}
& \epsilon_{a d}{ }^{e} \epsilon_{n}{ }^{a d}=2 \delta_{n}{ }^{e} \\
& \epsilon_{a b}{ }^{e} \epsilon_{n}{ }^{a d}=\delta_{b}{ }^{d} \delta_{n}{ }^{e}-\delta_{b n} \delta^{d e}
\end{aligned}
$$

where $\epsilon_{n}{ }^{a d}$ is the transpose of $\epsilon_{a d}^{n}$ in the $S U(2)$ metric. This yields, using the property $\mathrm{B}_{a b}^{c}=\mathrm{B}_{[a b]}^{c}$,

$$
0=\epsilon_{n}^{a d} H_{b c a d}=4 \delta_{n[b} \mathrm{B}_{c] a d} \delta^{a d}+2 \mathrm{~B}_{n c b}-2 \mathrm{~B}_{n b c}+2 \mathrm{~B}_{b c n}
$$

Permuting the free indices in Eq. (2.26) and adding the resulting equations now leads to

$$
0=\delta_{n[b} \mathrm{B}_{c] a d} \delta^{a d}+\mathrm{B}_{b c n}
$$

This algebraic relation implies 


$$
\mathrm{B}_{b c n}=\delta_{n b} v_{c}-\delta_{n c} v_{b}
$$

for some arbitrary constants $v_{c}$. The expression (2.28) is easily checked to satisfy Eq. (2.22) as well as the required properties

$$
\begin{aligned}
& \mathrm{B}_{b c n}=2 \delta_{n[b} v_{c]}=\mathrm{B}_{[b c] n}, \\
& \mathrm{~B}_{[b c}{ }^{n} \mathrm{~B}_{d] n}{ }^{a}=2 \delta_{[b}{ }^{n} v_{c} \delta_{d]}{ }^{a} v_{n}-2 \delta_{[b}{ }^{n} v_{c} v_{d]} \delta_{n}{ }^{a}=4 v_{[b} v_{c} \delta_{d]}{ }^{a}=0
\end{aligned}
$$

Thus, from gauge invariance, the algebraic condition (2.22) fixes the auxiliary structure constants (2.28) which appear in the field strength (2.8), the Lagrangian (2.9), and the gauge symmetry (2.10) of the theory.

These structure constants define an auxiliary Lie algebra multiplication

$$
\left[\mathbf{e}_{a}, \mathbf{e}_{b}\right]_{B}=\mathrm{B}_{a b} \mathbf{e}_{c}=2 v_{[b} \mathbf{e}_{a]}
$$

in terms of the $S U(2)$ basis vectors $\mathbf{e}_{a}$. The auxiliary multiplication is related to $S U(2)$ multiplication,

$$
\left[\mathbf{e}_{a}, \mathbf{e}_{b}\right]_{B}=\left[\left[\mathbf{e}_{a}, \mathbf{e}_{b}\right], \mathbf{v}\right]
$$

using the Lie-algebra vector $\mathbf{v}=v_{b} \delta^{b a} \mathbf{e}_{a}$ determined by the constants $v_{b}$. This relationship (2.32) directly follows from expressing the auxiliary structure constants in terms of the $S U(2)$ structure constants

$$
\epsilon_{a b}^{e} \epsilon_{e d}^{c} \delta^{d n} v_{n}=2 \delta_{[a}^{n} \delta_{b]}^{c} v_{n}=2 v_{[a} \delta_{b]}^{c}=\mathrm{B}_{a b}^{c}
$$

with the use of the $S U(2)$ identity (2.25) and some index rearrangements.

The structure of the auxiliary multiplication can be understood as follows. Fix two linearly independent Lie-algebra vectors $\mathbf{w}_{1}=w_{1}^{a} \mathbf{e}_{a}$ and $\mathbf{w}_{2}=w_{2}^{a} \mathbf{e}_{a}$ such that $w_{1}^{a} v_{a}=0=$ $w_{2}^{a} v_{a}$, so the vectors $\left\{\mathbf{w}_{1}, \mathbf{w}_{2}, \mathbf{v}\right\}$ provide a Lie algebra basis with $\mathbf{v}$ orthogonal to $\mathbf{w}_{1}$ and $\mathbf{w}_{2}$ in the $S U(2)$ Killing metric. From Eq. (2.31) the auxiliary multiplication of this basis is given by 


$$
\begin{aligned}
& {\left[\mathbf{w}_{1}, \mathbf{w}_{2}\right]_{B}=2 w_{1}^{a} w_{2}^{b} v_{[b} \mathbf{e}_{a]}=0} \\
& {\left[\mathbf{w}_{1}, \mathbf{v}\right]_{B}=2 w_{1}^{a} \delta^{c b} v_{c} v_{[b} \mathbf{e}_{a]}=\mathbf{w}_{1}|\mathbf{v}|^{2}} \\
& {\left[\mathbf{w}_{2}, \mathbf{v}\right]_{B}=2 w_{2}^{a} \delta^{c b} v_{c} v_{[b} \mathbf{e}_{a]}=\mathbf{w}_{2}|\mathbf{v}|^{2}}
\end{aligned}
$$

where $|\mathbf{v}|^{2}=\delta^{a b} v_{a} v_{b}$ is the $S U(2)$ norm squared of $\mathbf{v}$. This multiplication structure represents a three-dimensional nilpotent Lie algebra [⿴囗⿰丿㇄口] which is the semi-direct product of the one-dimensional Lie algebra spanned by $\mathbf{v}$ with the two-dimensional abelian Lie algebra spanned by $\mathbf{w}_{1}$ and $\mathbf{w}_{2}$, where $\frac{1}{|\mathbf{v}|^{2}} \mathbf{v}$ acts by multiplication as an identity element on $\mathbf{w}_{1}$ and $\mathbf{w}_{2}$.

\section{B. Features}

The variation of the Lagrangian (2.9) as assembled from Eqs. (2.11) to (2.13) yields the field equations for $A_{\mu}^{a}$,

$$
E_{\mu}^{a}=2 \epsilon_{\mu}{ }^{\sigma \nu}\left(\partial_{\sigma} K_{\nu}^{a}+\epsilon_{b c}^{a} A_{\sigma}^{b} K_{\nu}^{c}+\frac{1}{2} \mathrm{~B}_{b c}{ }^{a} K_{\sigma}^{b} K_{\nu}^{c}\right)=0
$$

The fields $A_{\mu}^{a}$ appear in $E_{\mu}^{a}$ nonpolynomially through the field strengths $K_{\mu}^{a}$, producing a novel form of nonlinear coupling of the fields, which is controlled by the auxiliary structure constants $\mathrm{B}_{b c}{ }^{a}$ together with the $S U(2)$ structure constants $\epsilon_{b c}{ }^{a}$.

The field strengths have two basic properties. First, the $S U(2)$ Bianchi identity $0=$ $\partial^{\mu} F_{\mu}^{a}+\epsilon_{b c}^{a} A_{\mu}^{b} F_{\nu}^{c} \eta^{\mu \nu}$ leads to the differential identity on $K_{\mu}^{a}$,

$$
\partial^{\mu} K_{\mu}^{a}+\left(\epsilon_{b c}^{a} A_{\mu}^{b} K_{\nu}^{c}+\mathrm{B}_{d c}^{a} K_{\mu}^{d} K_{\nu}^{c}\right) \eta^{\mu \nu}=\frac{1}{2} \mathrm{~B}_{d c}^{a} E_{\mu}^{d} A_{\nu}^{c} \eta^{\mu \nu}
$$

where $\partial^{\mu}=\eta^{\mu \nu} \partial_{\nu}$. Consequently, for solutions of the field equations, $K_{\mu}^{a}$ satisfies $S U(2)$ field equations of the form

$$
\begin{aligned}
& \eta^{\mu \nu}\left(\partial_{\mu} K_{\nu}^{a}+\epsilon_{b c}{ }^{a} A_{\mu}^{b} K_{\nu}^{c}\right)=-\mathrm{B}_{d c}^{a} K_{\mu}^{d} K_{\nu}^{c} \eta^{\mu \nu} \\
& \epsilon_{\mu}{ }^{\sigma \nu}\left(\partial_{\sigma} K_{\nu}^{a}+\epsilon_{b c}{ }^{a} A_{\sigma}^{b} K_{\nu}^{c}\right)=-\frac{1}{2} \mathrm{~B}_{b c}{ }^{a} K_{\sigma}^{b} K_{\nu}^{c} \epsilon_{\mu}{ }^{\sigma \nu}
\end{aligned}
$$


involving scalar and vector source terms generated quadratically from $K_{\mu}^{a}$. Second, under the gauge symmetry $(2.10)$ on $A_{\mu}^{a}$, the field strengths $K_{\mu}^{a}$ have the transformation

$$
\delta K_{\mu}^{a}=\epsilon_{b c}^{a} K_{\mu}^{b} \xi^{c}+\frac{1}{2}\left(Y^{-1} E\right)_{e \mu}^{a} \xi^{e}
$$

where $\left(Y^{-1} E\right)_{e \mu}^{a}=Y_{\mu b}^{-1 a \nu} E_{\nu}^{c} \mathrm{~B}_{c e}^{b}$. For solutions of the field equations, the form of Eq. (2.41) represents an infinitesimal rotation of $K_{\mu}^{a}$ as an $S U(2)$ vector, and so $S U(2)$ invariants constructed from $K_{\mu}^{a}$ yield gauge symmetry invariants in the theory.

The gauge symmetry of the theory has a closed commutator structure on solutions of the field equations. From Eqs. (2.10) and (2.41), calculating the commutator of two infinitesimal gauge transformations $\delta_{1} A_{\mu}^{a}$ and $\delta_{2} A_{\mu}^{a}$ involving sets of arbitrary functions $\xi_{1}^{a}$ and $\xi_{2}^{a}$ yields

$$
\left[\delta_{1}, \delta_{2}\right] A_{\mu}^{a}=\delta_{3} A_{\mu}^{a}+\mathrm{B}^{a}{ }_{c[d}\left(Y^{-1} E\right)_{e] \mu}^{c} \xi_{2}^{e} \xi_{1}^{d}
$$

where the infinitesimal gauge transformation $\delta_{3} A_{\mu}^{a}$ involves the set of functions $\xi_{3}^{c}=\epsilon_{e d}{ }^{c} \xi_{2}^{e} \xi_{1}^{d}$. Since $\left[\delta_{1}, \delta_{2}\right] A_{\mu}^{a}=\delta_{3} A_{\mu}^{a}$ on solutions of the field equations, the commutator structure is given by structure constants of the Lie algebra $S U(2)$. Hence, the gauge symmetry generates a closed group of finite gauge transformations on solutions $A_{\mu}^{a}$.

Associated to the gauge symmetry group are conserved currents $\partial^{\mu} J_{\mu}^{a}=0$ given by

$$
J_{\mu}^{a}=\epsilon_{\mu}^{\sigma \nu} \partial_{\sigma} K_{\nu}^{a}
$$

in terms of the field strengths $K_{\mu}^{a}$ for solutions of the field equations. If we consider twodimensional hypersurfaces $\Sigma$ of the underlying three-dimensional manifold on which the fields $A_{\mu}^{a}$ are defined, the flux of the currents $J_{\mu}^{a}$ on a given $\Sigma$ defines internal charges carried by the fields on $\Sigma$. We can evaluate these charges by the surface integral

$$
Q^{a}=\int_{\Sigma} t^{\mu} J_{\mu}^{a}
$$

where $t^{\mu}$ is a unit normal to $\Sigma$ and the integral is understood to use the induced volume element $t^{\mu} \epsilon_{\mu \sigma \tau}$ on $\Sigma$. If $\Sigma$ has topology $R^{2}$, then we can express $Q^{a}$ by a line integral

$$
Q^{a}=\int_{\Sigma} t^{\mu} J_{\mu}^{a}=\oint_{C} s^{\mu} K_{\mu}^{a}
$$


where $C$ is the boundary at infinity on $\Sigma$ and $s^{\mu}$ is the unit tangent to $C$, in a clockwise orientation. When there is no current flow across $C$, the charges defined by $Q^{a}$ are conserved, $t^{\mu} \partial_{\mu} Q^{a}=0$

The charges $Q^{a}$ transform as $S U(2)$ vectors

$$
\delta Q^{a}=\epsilon_{b c}{ }^{a} Q^{b} \xi^{c}
$$

under the gauge symmetry (2.10) and (2.41) if the functions $\xi^{c}$ are constant on $C$. Applying Noether's theorem to this restricted gauge symmetry yields a direct derivation of the charges $Q^{a}$ from the Lagrangian (2.9) of the theory.

A conserved, gauge invariant energy-momentum tensor $\partial^{\mu} T_{\mu \nu}=0$ can be derived from the Lagrangian by varying the inverse metric $\eta^{\mu \nu}$ in $L \epsilon_{\sigma \beta \alpha}$ as follows,

$$
T_{\mu \nu}=\epsilon^{\sigma \beta \alpha} \delta\left(L \epsilon_{\sigma \beta \alpha}\right) / \delta \eta^{\mu \nu}=\delta_{a b}\left(K_{\mu}^{a} K_{\nu}^{b}-\frac{1}{2} \eta_{\mu \nu} \eta^{\sigma \tau} K_{\sigma}^{a} K_{\tau}^{b}\right)
$$

after some cancellations of terms. Conservation of $T_{\mu \nu}$ can then be shown from the covariance property of the Lagrangian, $\delta L=\mathcal{L}_{\zeta} L$ under simultaneous variations of the inverse metric $\delta \eta^{\mu \nu}=\mathcal{L}_{\zeta} \eta^{\mu \nu}$ and the volume form $\delta \epsilon_{\sigma \beta \alpha}=\mathcal{L}_{\zeta} \epsilon_{\sigma \beta \alpha}$ as well as the fields $\delta A_{\mu}^{a}=\mathcal{L}_{\zeta} A_{\mu}^{a}$ where $\mathcal{L}_{\zeta}$ is the Lie derivative generated by an arbitrary vector field $\zeta^{\sigma}$. Gauge invariance of $T_{\mu \nu}$ follows directly from the transformation property $\delta K_{\mu}^{a}=\epsilon_{b c}{ }^{a} K_{\mu}^{b} \xi^{c}$ of the field strengths under the gauge symmetry (2.10) and (2.41) on solutions of the field equations.

Conserved currents are obtained from $T_{\mu \nu}$ by contracting with a Killing vector field $\zeta^{\nu}$ of the metric, yielding the current $T_{\mu \nu} \zeta^{\nu}$, where $\partial^{(\mu} \zeta^{\nu)}=0$. If we consider two-dimensional hypersurfaces $\Sigma$ as above, these conserved currents then define gauge invariant fluxes of energy-momentum and stress carried by the fields $A_{\mu}^{a}$ on a given $\Sigma$, with $\zeta^{\nu}$ being respectively a time translation isometry and a space translation isometry. (Fluxes of angular momentum are defined with $\zeta^{\nu}$ being a rotation or boost isometry). The fluxes are given by the surface integral

$$
Q_{\zeta}=\int_{\Sigma} t^{\mu} T_{\mu \nu} \zeta^{\nu}
$$


where $t^{\mu}$ is a unit normal to $\Sigma$ and the integral uses the induced volume element $t^{\mu} \epsilon_{\mu \sigma \tau}$ on $\Sigma$.

\section{Gauge Covariant Formulation}

The theory has a natural formulation using a field variable $\mathbf{A}_{\mu}=A_{\mu}^{a} \mathbf{e}_{a}$ which is an $S U(2)$ Lie-algebra valued vector field. The $S U(2)$ covariant derivative and curvature associated to $\mathbf{A}_{\mu}$ as a connection geometrically in $S U(2)$ Yang-Mills theory enter directly into the formulation.

We start from the $S U(2)$ Lie bracket defined by

$$
[\phi, \psi]=\epsilon_{b c}{ }^{a} \phi^{b} \psi^{c} \mathbf{e}_{a}
$$

where $\phi=\phi^{b} \mathbf{e}_{b}$ and $\psi=\psi^{b} \mathbf{e}_{b}$ are arbitrary $S U(2)$ Lie-algebra valued fields. From the relation (2.33) for the auxiliary structure constants $\mathrm{B}_{a b}{ }^{c}$ expressed in terms of the $S U(2)$ structure constants $\epsilon_{a b}{ }^{c}$, we then have

$$
\begin{aligned}
& \mathrm{B}_{a b}{ }^{c} \phi^{a} \psi^{b} \mathbf{e}_{c}=[[\phi, \psi], \mathbf{v}] \\
& \mathrm{B}^{a}{ }_{d c} \phi^{d} \psi^{c} \mathbf{e}_{c}=-[\phi,[\psi, \mathbf{v}]]
\end{aligned}
$$

which allows all the structures in the theory involving $\mathrm{B}_{a b}{ }^{c}$ and $\mathrm{B}^{a}{ }_{b c}$ to be formulated using the $S U(2)$ Lie bracket [, ] and the $S U(2)$ vector $\mathbf{v}=v_{b} \delta^{b a} \mathbf{e}_{a}$.

Next we utilize $\mathbf{A}_{\mu}$ to define the $S U(2)$ covariant derivative

$$
D_{\mu} \phi=\partial_{\mu} \phi+\left[\mathbf{A}_{\mu}, \phi\right]=\partial_{\mu} \phi^{a} \mathbf{e}_{a}+\epsilon_{b c}{ }^{a} A_{\mu}^{b} \phi^{c} \mathbf{e}_{a}
$$

The curvature of the covariant derivative is defined through the relation

$$
D_{[\mu} D_{\nu]} \phi=\left[\mathbf{F}_{\mu \nu}, \phi\right]=\epsilon_{b c}{ }^{a} F_{\mu \nu}^{b} \phi^{c} \mathbf{e}_{a}
$$

To now proceed with the formulation, we introduce the field strength $\mathbf{K}_{\mu}=K_{\mu}^{a} \mathbf{e}_{a}$ for the theory by the algebraic relation 


$$
\mathbf{K}_{\mu}+\epsilon_{\mu}{ }^{\nu \sigma}\left[\mathbf{K}_{\nu},\left[\mathbf{A}_{\sigma}, \mathbf{v}\right]\right]=\mathbf{F}_{\mu}
$$

where $\mathbf{F}_{\mu}=\epsilon_{\mu}{ }^{\nu \sigma} \mathbf{F}_{\nu \sigma}$ is the dual of the $S U(2)$ curvature tensor. In terms of $\mathbf{K}_{\mu}$ and $\mathbf{A}_{\mu}$, the Lagrangian is given by

$$
L=\eta^{\mu \nu}\left(\mathbf{K}_{\mu}, \mathbf{K}_{\nu}\right)+\epsilon^{\sigma \tau \nu}\left(\left[\mathbf{K}_{\sigma}, \mathbf{K}_{\tau}\right],\left[\mathbf{A}_{\nu}, \mathbf{v}\right]\right)
$$

where ( , ) is the $S U(2)$ Killing metric.

The field equations derived from $L$ by varying $\mathbf{A}_{\mu}$ are given by

$$
2 \epsilon_{\mu}{ }^{\sigma \tau}\left(D_{\sigma} \mathbf{K}_{\tau}+\frac{1}{2}\left[\left[\mathbf{K}_{\sigma}, \mathbf{K}_{\tau}\right], \mathbf{v}\right]\right)=0
$$

and the differential identity satisfied by $\mathbf{K}_{\mu}$ for solutions of the field equations is given by

$$
\eta^{\sigma \tau}\left(D_{\sigma} \mathbf{K}_{\tau}-\left[\mathbf{K}_{\sigma},\left[\mathbf{K}_{\tau}, \mathbf{v}\right]\right]\right)=0
$$

The gauge symmetry on solutions of the field equations is given by the infinitesimal transformations

$$
\begin{aligned}
\delta \mathbf{A}_{\mu} & =D_{\mu} \xi+\left[\mathbf{K}_{\mu},[\mathbf{v}, \xi]\right] \\
\delta \mathbf{K}_{\mu} & =\left[\mathbf{K}_{\mu}, \xi\right]
\end{aligned}
$$

where $\xi$ is an $S U(2)$ Lie-algebra valued arbitrary function.

Exponentiating the transformations (2.58) and (2.59) generates finite gauge symmetry transformations in the theory as follows. First, we express commutators involving $\xi$ by the linear operator $\operatorname{ad}_{\xi}=[\cdot, \xi]$ which acts on $S U(2)$ vectors. We also use the linear operator $\mathrm{P}_{\perp \xi}=-\frac{1}{|\xi|^{2}}\left(\mathrm{ad}_{\xi}\right)^{2}$ which acts as the projection onto $S U(2)$ vectors orthogonal to $\xi$ in the $S U(2)$ Killing metric, where $(\xi, \xi)=|\xi|^{2}$. Then, calculating $\exp (\delta)$ with the operator $\delta$ given by the infinitesimal transformations (2.58) and (2.59) on $\mathbf{A}_{\mu}$ and $\mathbf{K}_{\mu}$ leads to the finite transformations

$$
\begin{aligned}
& \mathbf{A}_{\mu} \rightarrow R_{\xi} \mathbf{A}_{\mu}+R_{\xi}^{\prime} \partial_{\mu} \xi+\left(R_{\xi}^{\prime} \mathbf{K}_{\mu}, \mathbf{v}\right) \xi-\left(\mathbf{K}_{\mu}, \xi\right) R_{\xi}^{\prime} \mathbf{v} \\
& \mathbf{K}_{\mu} \rightarrow R_{\xi} \mathbf{K}_{\mu}
\end{aligned}
$$


where $R_{\xi}=\exp \left(\operatorname{ad}_{\xi}\right)$ is a rotation generated by $\xi$ on $S U(2)$ vectors, and $R_{\xi}^{\prime}=\mathbf{1}-\mathrm{P}_{\perp \xi}-$ $\frac{1}{|\xi|^{2}} \operatorname{ad}_{\xi}\left(R_{\xi}-\mathbf{1}\right)$ is a related transformation on $S U(2)$ vectors. Explicitly,

$$
\begin{aligned}
& R_{\xi}=\mathbf{1}+(\sin |\xi|) \operatorname{ad}_{\hat{\xi}}-(\mathbf{1}-\cos |\xi|) \mathrm{P}_{\perp \hat{\xi}} \\
& R_{\xi}^{\prime}=\mathbf{1}+\frac{1}{|\xi|}(\mathbf{1}-\cos |\xi|) \operatorname{ad}_{\hat{\xi}}-\left(\mathbf{1}-\frac{1}{|\xi|} \sin |\xi|\right) \mathrm{P}_{\perp \hat{\xi}}
\end{aligned}
$$

in terms of the unit vector $\hat{\xi}=\frac{1}{|\xi|} \xi$. (The relation between $R_{\xi}$ and $R_{\xi}^{\prime}$ is expressed by [5] the composition formula of rotations $R_{\xi_{1}} R_{\xi_{2}}=R_{\xi_{3}}$, where $\xi_{3}=\xi_{1}+R_{\xi_{1}}^{\prime-1} \xi_{2}$.)

The transformations (2.60) and (2.61) represent a closed group of finite gauge symmetries with the novel feature that the group acts nonlinearly on $\mathbf{A}_{\mu}$ but linearly on $\mathbf{K}_{\mu}$, where $\mathbf{K}_{\mu}$ is a nonpolynomial algebraic expression in terms of $\mathbf{A}_{\mu}$ determined by Eq. (2.54). Algebraically, the group structure of the finite gauge symmetries is isomorphic to the exponential of the $S U(2)$ Lie algebra structure (2.42) of the infinitesimal gauge symmetries (2.10).

The appearance of $\mathbf{v}$ in the field equations and gauge symmetry of the theory defines a preferred vector in the $S U(2)$ Lie algebra of the gauge group. Consequently, the theory lacks symmetry invariance under rigid $S U(2)$ rotations on the field variable

$$
\mathbf{A}_{\mu} \rightarrow R \mathbf{A}_{\mu}
$$

where $R=\exp \left(\operatorname{ad}_{\xi}\right)$ is a constant transformation generated by an arbitrary $S U(2)$ vector $\xi$, with $\partial_{\mu} \xi=0$.

The effect of a transformation (2.64) in the theory is to rotate the preferred $S U(2)$ vector

$$
\mathbf{v} \rightarrow R \mathbf{v}
$$

Thus, the direction of $\mathbf{v}$ can be changed arbitrarily under field redefinitions given by the transformations (2.64).

When $\mathbf{v}=0$, the $S U(2)$ symmetry invariance (2.64) is restored, and the theory then reduces to $S U(2)$ Yang-Mills theory. 


\section{EXTENSION TO OTHER GAUGE GROUPS}

We now carry out the extension from an $S U(2)$ gauge group to a general nonabelian gauge group for the Yang-Mills theory generalization in Sec. II]. Since the most general gauge group for Yang-Mills theory is required to be semisimple [5], we only need to consider semisimple gauge groups for the generalization. The structure of these groups is characterized by the Lie algebra of the group being a product of abelian Lie algebras and nonabelian simple Lie algebras [6].

We start with an arbitrary simple Lie algebra for the gauge group Lie algebra, with multiplication structure constants $\mathrm{C}_{b c}{ }^{a}$ and Killing metric components $k_{a b}=-\frac{1}{2} \mathrm{C}_{a d}{ }^{e} \mathrm{C}_{b e}{ }^{d}$ in a fixed Lie algebra basis. We introduce structure constants $\mathrm{B}_{b c}{ }^{a}$ defining an auxiliary Lie algebra multiplication which is related to the gauge group Lie algebra multiplication later through an algebraic condition imposed by gauge invariance. Now, setting the number of fields $A_{\mu}^{a}$ to match the dimension of the gauge group Lie algebra, we use $\mathrm{C}_{b c}{ }^{a}$ and $k_{a b}$ to replace the $S U(2)$ structure constants $\epsilon_{b c}{ }^{a}$ and Killing metric components $\delta_{a b}$ in the form of the field strengths $K_{\mu}^{a}$, the Lagrangian $L$, and the gauge symmetry $\delta A_{\mu}^{a}$ of the $S U(2)$ theory given in Eqs. (2.8) to (2.10).

Gauge invariance requires that the Lagrangian $L$ vary into a complete divergence under the gauge symmetry $\delta A_{\mu}^{a}$. By the same calculations as followed in the $S U(2)$ case, after using the antisymmetry property $\mathrm{B}_{b c}{ }^{e}=\mathrm{B}_{[b c]}{ }^{e}$ and Jacobi property $\mathrm{B}_{[b c}{ }^{e} \mathrm{~B}_{d] e n}=0$ of the auxiliary structure constants in addition to the Jacobi property $\mathrm{C}_{[b c}{ }^{e} \mathrm{C}_{d] e n}=0$ and antisymmetry property $\mathrm{C}_{[b c}{ }^{e} k_{d] e}=\mathrm{C}_{b c}{ }^{e} k_{d e}$ of the gauge group structure constants, we find $\delta L$ is a complete divergence up to the term $\epsilon^{\nu \sigma \tau} K_{\nu}^{b} K_{\sigma}^{c} A_{\tau}^{a} \xi^{d} H_{b c a d}$ where

$$
H_{b c a d}=2 \mathrm{C}_{a[b}{ }^{e} \mathrm{~B}_{|e| c] d}-2 \mathrm{C}_{d[b}{ }^{e} \mathrm{~B}_{|e| c] a}+\mathrm{C}_{a d}{ }^{e} \mathrm{~B}_{b c e}
$$

with $\mathrm{B}_{b c e}=\mathrm{B}_{b c}{ }^{a} k_{a e}$. Thus, similarly to the $S U(2)$ case, we must have

$$
0=H_{b c a d}
$$

which is an algebraic condition relating the structure constants $\mathrm{C}_{b c}{ }^{a}$ and $\mathrm{B}_{b c}{ }^{a}$. 
Condition (3.2) can be solved, as shown in App. A, yielding

$$
\mathrm{B}_{b c a}=2 V_{e[b} \mathrm{C}_{c] a}^{e}
$$

where $V_{e b}=V_{[e b]}$ represent arbitrary constants. This expression for $\mathrm{B}_{b c a}$ does not automatically satisfy the Jacobi property $\mathrm{B}_{[b c}{ }^{n} \mathrm{~B}_{d] n}{ }^{a}=0$ except in the $S U(2)$ case as follows. By the $S U(2)$ identities (2.24) and (2.25), $V_{e b}$ can be expressed equivalently as $\epsilon_{e b}{ }^{d} v_{d}$ in terms of $v_{d}=\frac{1}{2} \epsilon_{d}^{e b} V_{e b}$, so the expression $\mathrm{B}_{b c a}=2 V_{e[b} \epsilon_{c] a}{ }^{e}$ has the form

$$
\mathrm{B}_{b c a}=2 \epsilon_{e[b}{ }^{d} \epsilon_{c] a}{ }^{e} v_{d}=\epsilon_{b c}{ }^{e} \epsilon_{a e}{ }^{d} v_{d}
$$

The Jacobi property for $\mathrm{B}_{b c a}$ then becomes a consequence of the $S U(2)$ identities, after some manipulations shown by Eqs. (2.30) and (2.33).

Other than in the $S U(2)$ case, the Jacobi property for $\mathrm{B}_{b c a}$ must be imposed as an extra condition

$$
0=\mathrm{B}_{[b c}^{n} \mathrm{~B}_{d] n a}=\frac{4}{3} k^{m n}\left(V_{e[b} \mathrm{C}_{c] n}{ }^{e} V_{p[d} \mathrm{C}_{m] a}{ }^{p}+V_{e[d} \mathrm{C}_{b] n}{ }^{e} V_{p[c} \mathrm{C}_{m] a}{ }^{p}+V_{e[c} \mathrm{C}_{d] n}{ }^{e} V_{p[b} \mathrm{C}_{m] a}{ }^{p}\right)
$$

which constitutes an algebraic equation on $V_{e b}$. The solutions of this equation determine the form for $\mathrm{B}_{b c a}$ necessary for gauge invariance of the Yang-Mills theory generalization with a general nonabelian simple gauge group.

Since $V_{e b}$ enters Eq. (3.5) quadratically, finding the complete solution is a difficult algebraic problem. A natural possibility is to consider the expression $V_{e b}=\mathrm{C}_{e b}{ }^{d} v_{d}$ of the same form as works in the $S U(2)$ case, leading to $\mathrm{B}_{b c a}=\mathrm{C}_{b c}{ }^{e} \mathrm{C}_{a e}{ }^{d} v_{d}$ similarly to Eq. (3.4). We find the following results for the cases of $S U(n)$ and $S O(n)$ gauge groups. Because of the Lie algebra isomorphism $S U(2) \simeq S O(3)$, the $S O(3)$ case is the same as the $S U(2)$ case. When the expression for $\mathrm{B}_{b c a}$ is extended from the $S U(2)$ case to the $S O(n)$ case, condition (3.5) is satisfied for $n=4$ as a consequence of the Lie algebra isomorphism between $S O(4)$ and a real form of the complexified product $S U(2) \times S U(2)$. However, condition (3.5) fails to be satisfied in any other $S O(n)$ case. The condition also fails to be satisfied when the expression for $\mathrm{B}_{b c a}$ is extended to the $S U(n)$ case for any $n \geq 3$. Hence, among the $S U(n)$ 
and $S O(n)$ cases, when a relation of the same form between the gauge group structure constants and auxiliary structure constants as holds in the $S U(2)$ case is used, the Yang-Mills theory generalization works only in the case of an $S O(4)$ gauge group.

We now return to the general case of a nonabelian simple gauge group. The simplest alternative possibility to consider is the elementary expression $V_{e b}=2 u_{[e} v_{b]}$ for some constants $u_{e}$ and $v_{b}$. As shown in App. A, the condition (3.5) on $V_{e b}$ is satisfied by having

$$
\mathrm{C}_{d c}{ }^{a} u^{d} v^{c}=0
$$

where $u^{d}=k^{d e} u_{e}$ and $v^{c}=k^{c e} v_{e}$ determine Lie algebra vectors that commute in the gauge group Lie algebra. Algebraically, this requires the gauge group Lie algebra to have a commutative subalgebra of dimension at least two, as met by any simple Lie algebra other than $S U(2)$. Hence, for these simple Lie algebras, the expression

$$
\mathrm{B}_{b c a}=2 v_{[b} \mathrm{C}_{c] a}{ }^{e} u_{e}-2 u_{[b} \mathrm{C}_{c] a}{ }^{e} v_{e}
$$

determined by Eqs. (3.3) and (3.6) satisfies the Jacobi property (3.5). With the relation (3.7) between the gauge group structure constants and auxiliary structure constants, the Yang-Mills theory generalization works for all nonabelian simple gauge groups other than $S U(2)$.

The Yang-Mills theory generalization also works if the relation (3.7) is generalized to a sum of similar expressions using pairs of Lie algebra vectors that all commute in the simple gauge group Lie algebra. (In particular, for the case of $S U(n)$, the number of linearly independent commuting vectors is $n-1$, and for the case of $S O(n)$, the number is $\frac{1}{2} n$ if $n$ is even and $\frac{1}{2}(n-1)$ if $n$ is odd.) The same relation can used more generally with semisimple gauge groups, where the Lie algebra vectors lie in different simple subalgebras of the semi-simple gauge group Lie algebra. 


\section{A. Gauge covariant formulation of the extension}

Relation (3.7) between the gauge group structure constants $\mathrm{C}_{b c}{ }^{a}$ and the auxiliary structure constants $\mathrm{B}_{b c}{ }^{a}$ differs from the $S U(2)$ case, giving a somewhat different gauge covariant structure for the resulting Yang-Mills theory generalization. We now outline this structure.

We fix Lie algebra basis vectors $\mathbf{e}_{a}$ associated to the gauge group structure constants,

$$
\left[\mathbf{e}_{a}, \mathbf{e}_{b}\right]=\mathrm{C}_{a b}{ }^{c} \mathbf{e}_{c}
$$

and introduce Lie algebra vectors $\mathbf{u}=u^{a} \mathbf{e}_{a}$ and $\mathbf{v}=v^{b} \mathbf{e}_{b}$ defined from the constants $u^{a}=$ $\delta^{a c} u_{c}, v^{b}=\delta^{b d} v_{d}$. Using these vectors we define the linear map

$$
V(\phi)=(\mathbf{u}, \phi) \mathbf{v}-(\mathbf{v}, \phi) \mathbf{u}
$$

on Lie-algebra vectors $\phi=\phi^{a} \mathbf{e}_{a}$. All structure involving the auxiliary structure constants can now be expressed completely in terms of the map (3.9) and the Lie bracket (3.8).

We introduce $\mathbf{A}_{\mu}=A_{\mu}^{a} \mathbf{e}_{a}$ as the field variable for the gauge covariant formulation of the theory. We also use the covariant derivative defined in terms of $\mathbf{A}_{\mu}$ by

$$
D_{\mu}=\partial_{\mu}+\left[\mathbf{A}_{\mu}, \cdot\right]
$$

along with the dual of the curvature tensor of this derivative operator, defined as

$$
\mathbf{F}_{\sigma}=\epsilon_{\sigma}^{\mu \nu}\left(\partial_{\mu} \mathbf{A}_{\nu}+\frac{1}{2}\left[\mathbf{A}_{\mu}, \mathbf{A}_{\nu}\right]\right)
$$

The formulation of the field strengths $\mathbf{K}_{\mu}=K_{\mu}^{a} \mathbf{e}_{a}$ in the theory is given by the algebraic relation

$$
\mathbf{K}_{\mu}-\epsilon_{\mu}{ }^{\nu \sigma}\left(V\left(\left[\mathbf{K}_{\nu}, \mathbf{A}_{\sigma}\right]\right)+\left[V\left(\mathbf{K}_{\nu}\right), \mathbf{A}_{\sigma}\right]\right)=\mathbf{F}_{\mu}
$$

The Lagrangian of the theory has the formulation

$$
L=\eta^{\sigma \tau}\left(\mathbf{K}_{\sigma}, \mathbf{K}_{\tau}\right)+2 \epsilon^{\mu \nu \tau}\left(\left[V\left(\mathbf{K}_{\mu}\right), \mathbf{K}_{\nu}\right], \mathbf{A}_{\tau}\right)
$$


where $($,$) is the Killing metric in terms of the gauge group structure constants, such that$ $\left(\mathbf{e}_{a}, \mathbf{e}_{b}\right)=k_{a b}$. The field equations for $\mathbf{A}_{\mu}$ from varying $L$ have the formulation

$$
0=2 \epsilon_{\mu}{ }^{\sigma \tau}\left(D_{\sigma} \mathbf{K}_{\tau}-\left[V\left(\mathbf{K}_{\sigma}\right), \mathbf{K}_{\tau}\right]\right)
$$

and the differential identity satisfied by the field strength for solutions of the field equations has the formulation

$$
0=\eta^{\sigma \tau}\left(D_{\sigma} \mathbf{K}_{\tau}+V\left(\left[\mathbf{K}_{\sigma}, \mathbf{K}_{\tau}\right]\right)-\left[V\left(\mathbf{K}_{\sigma}\right), \mathbf{K}_{\tau}\right]\right)
$$

The gauge symmetry on solutions of the field equations in the theory is given by infinitesimal transformations with the formulation

$$
\begin{aligned}
\delta \mathbf{A}_{\mu} & =D_{\mu} \xi+V\left(\left[\mathbf{K}_{\mu}, \xi\right]\right)-\left[V\left(\mathbf{K}_{\mu}\right), \xi\right] \\
\delta \mathbf{K}_{\mu} & =\left[\mathbf{K}_{\mu}, \xi\right]
\end{aligned}
$$

where $\xi$ is a Lie-algebra valued arbitrary function. These transformations have a closed commutator structure

$$
\begin{aligned}
{\left[\delta_{1} \mathbf{A}_{\mu}, \delta_{2} \mathbf{A}_{\mu}\right] } & =\delta_{3} \mathbf{A}_{\mu} \\
{\left[\delta_{1} \mathbf{K}_{\mu}, \delta_{2} \mathbf{K}_{\mu}\right] } & =\delta_{3} \mathbf{K}_{\mu}
\end{aligned}
$$

which is the same as the gauge group Lie alebra, with $\xi_{3}=\left[\xi_{1}, \xi_{2}\right]$. Thus, Lie algebra invariants constructed from $\mathbf{K}_{\mu}$ yield gauge symmetry invariants in the theory.

\section{DISCUSSION}

In this paper a class of new nonabelian gauge theories has been constructed for vector fields on three-dimensional manifolds. These theories describe a generalization of nonabelian Yang-Mills theory with a novel nonlinear gauge symmetry and field equations for threedimensional vector potential fields.

The new theories can be derived by a systematic generalization process starting from the linear gauge theory of vector potential fields given by abelian (linearized) Yang-Mills theory. 
The process consists of adding linear and higher order terms to the form of the abelian gauge symmetry while also adding quadratic and higher order terms to the form of the abelian field equations so as to maintain a gauge invariant action principle, with the condition of gauge invariance used as an equation to determine the allowed form of terms added order by order [1,7]. The linear order terms starting the process are provided by rigid symmetries special to abelian Yang-Mills fields $A_{\mu}^{a}$ in three dimensions

$$
\delta A_{\mu}^{a}=\left(\mathrm{C}_{b c}^{a} A_{\mu}^{b}+\mathrm{B}^{a b}{ }_{c} k_{b d} \epsilon_{\mu}{ }^{\sigma \nu} F_{\sigma \nu}^{d}\right) \xi^{c}
$$

which uses arbitrary constants $\mathrm{B}^{a b}{ }_{c}=\mathrm{B}^{[a b]}$, along with the structure constants $\mathrm{C}_{d c}{ }^{e}$ of the Lie algebra of any nonabelian Yang-Mills gauge group, the Killing metric $k_{b d}$ of this Lie algebra, the cross-product operator $\epsilon_{\mu}{ }^{\sigma \nu}$ on three-dimensional vectors, and an arbitrary rigid parameter, $\partial_{\mu} \xi^{c}=0$. For solutions $A_{\mu}^{a}$ of the abelian field equations, where $F_{\sigma \nu}^{d}$ is curl of $A_{\mu}^{a}$, the symmetries have a closed commutator structure $\left[\delta_{1} A_{\mu}^{a}, \delta_{2} A_{\mu}^{a}\right]=\delta_{3} A_{\mu}^{a}$ which involves $\xi^{c}, \mathrm{C}_{a b}{ }^{c}$ and $\mathrm{B}^{a b}{ }_{c}$. The existence of these symmetries is limited to three dimensions because of the dependence on the cross-product operator. Completing the generalization process with the linear terms given by Eq. (4.1) leads to successively higher order terms, and fixes $\mathrm{B}^{a b}{ }_{c}$ in terms of structure constants of an auxiliary Lie algebra related to the Lie algebra of the Yang-Mills gauge group, producing the striking nonlinearity in the form for the gauge symmetry and field equations.

The generalization process can be carried out more broadly in three dimensions starting with the most general form for linear order terms and requiring the minimum number of derivatives in the form for higher order terms in the gauge symmetry and field equations. The outcome of the process leads directly to the new theories, as can be shown following methods developed in Ref. [7] This establishes a strong uniqueness result for the theories as nonlinear generalizations of abelian Yang-Mills theory in three dimensions.

Is there a simple underlying geometrical structure to the theories? Yang-Mills theory has a geometrical structure which is understood in terms of the vector potential as a connection on a fiber bundle. In the new theories, the vector potential appears to have a different 
geometrical role more general than a connection on a fiber bundle, which is somehow tied to the auxiliary Lie algebra related to the gauge group Lie algebra underlying the structure of the theories. Understanding this structure geometrically would be highly worthwhile.

Can the theories be extended to higher dimensions? The rigid symmetries (4.1) needed for the generalization of abelian Yang-Mills theory in three dimensions cannot be extended to abelian Yang-Mills fields in other dimensions. However, there are closely analogous symmetries of the linear gauge theory of abelian Yang-Mills fields and antisymmetric tensor fields in four (and higher) dimensions, which can be used to start the generalization process. The outcome of this process leads to a novel nonlinear generalization of four-dimensional Yang-Mills gauge theory with antisymmetric tensor fields. A full discussion of this new gauge theory is given in a forthcoming paper [8].

Investigation of these new gauge theories in three and four dimensions could well hold significant interest for many areas of physics and mathematics.

\section{APPENDIX A: RELATION BETWEEN THE AUXILIARY LIE ALGEBRA AND THE GAUGE GROUP LIE ALGEBRA}

We begin by solving condition (3.2) to obtain the relation (3.3) for $\mathrm{B}_{b c}{ }^{a}$ in terms of $\mathrm{C}_{b c}{ }^{a}$ for any semi-simple gauge group Lie algebra. Throughout we raise and lower indices on $\mathrm{B}_{b c}{ }^{a}$ and $\mathrm{C}_{b c}{ }^{a}$ by $k_{m n}$ and its inverse $k^{m n}$, and we freely use the antisymmetry $\mathrm{B}_{b c a}=\mathrm{B}_{[b c] a}$ and the complete antisymmetry $\mathrm{C}_{b c a}=\mathrm{C}_{[b c a]}$ which follows since $k_{m n}$ is the Killing metric with respect to $\mathrm{C}_{b c a}$.

To proceed, we contract $k^{c d}$ onto $H_{b c a d}=0$, yielding

$$
\mathrm{C}_{b}^{d c} \mathrm{~B}_{d c a}=\mathrm{C}_{a b}{ }^{d} \mathrm{~B}_{d c}{ }^{c}
$$

We next contract $\mathrm{C}_{a d}{ }^{n}$ onto $H_{b c a d}=0$. After some rearrangements of indices and use of the Jacobi property of $\mathrm{C}_{b c a}$, we obtain

$$
0=2 \mathrm{~B}_{n c d}+\mathrm{B}_{c b a} \mathrm{C}_{e}^{b a} \mathrm{C}_{n d}{ }^{e}+\mathrm{B}_{e b a} \mathrm{C}_{n}{ }^{b a} \mathrm{C}_{c d}{ }^{e}-\frac{1}{2} \mathrm{~B}^{b e}{ }_{d} \mathrm{C}_{b e}{ }^{a} \mathrm{C}_{a n c}
$$


Antisymmetrizing on the indices $n, c$ leads to

$$
\mathrm{B}_{n c d}=\frac{1}{2} \mathrm{~B}_{[n \mid b a} \mathrm{C}_{e}^{b a} \mathrm{C}_{\mid c] d}{ }^{e}-\frac{1}{2} \mathrm{~B}_{e b a} \mathrm{C}_{[n}{ }^{b a} \mathrm{C}_{c] d}{ }^{e}+\frac{1}{4} \mathrm{C}_{n c a} \mathrm{C}_{b e}{ }^{a} \mathrm{~B}^{b e}{ }_{d}
$$

We now rearrange the last term into the form $-\frac{1}{2} \mathrm{~B}_{a b}{ }^{b} \mathrm{C}_{[n \mid e}{ }^{a} \mathrm{C}_{c] d}{ }^{e}$ using the Jacobi property of $\mathrm{C}_{b c a}$ and the relation (A1). Finally, combining this term with the other terms in Eq. (A3) yields

$$
\mathrm{B}_{n c d}=\frac{1}{2}\left(\mathrm{~B}_{[n \mid b a} \mathrm{C}_{e}^{b a}-\mathrm{B}_{e b a} \mathrm{C}_{[n}^{b a}-\frac{1}{2} \mathrm{C}_{[n \mid e}{ }^{a} \mathrm{~B}_{a b}{ }^{b}\right) \mathrm{C}_{\mid c] d}{ }^{e}
$$

This relation indicates $\mathrm{B}_{n c d}$ has the form $2 V_{e[n} \mathrm{C}_{c] d}{ }^{e}$ where $4 V_{e n}$ is identified with the expression in parenthesis.

To complete the solution, we show that $H_{b c a d}=0$ is satisfied by $\mathrm{B}_{n c d}=2 V_{e[n} \mathrm{C}_{c] d}{ }^{e}$ with $V_{e n}=V_{[e n]}$ taken to be arbitrary. Substituting $\mathrm{B}_{n c d}$ into $H_{b c a d}$ yields

$$
H_{b c a d}=4 \mathrm{C}_{[a \mid[b}^{e} V_{c] n} \mathrm{C}_{\mid d] e^{n}}+4 \mathrm{C}_{[a \mid[b}{ }^{e} \mathrm{C}_{c] \mid d]}{ }^{n} V_{n e}+2 \mathrm{C}_{a d}{ }^{e} V_{n[c} \mathrm{C}_{b] e}{ }^{n}
$$

In Eq. (A5) the middle term vanishes since $\mathrm{C}_{[a \mid[b}{ }^{e} \mathrm{C}_{c] \mid d]}{ }^{n}$ is symmetric in the indices $e, n$ while $V_{n e}$ is antisymmetric. The first term in Eq. (A5) can be rearranged using the Jacobi property of $\mathrm{C}_{a b}{ }^{e}$ to yield $-2 \mathrm{C}_{a d}{ }^{e} V_{n[c} \mathrm{C}_{b] e}{ }^{n}$ which cancels the last term in Eq. (A5). This demonstrates $H_{b c a d}=0$.

We now show that the Jacobi property (3.5) on $\mathrm{B}_{b c d}=2 V_{e[b} \mathrm{C}_{c] d} e^{e}$ is satisfied by $V_{e b}=$ $2 u_{[e} v_{b]}$ where $u_{e}$ and $v_{b}$ commute in the gauge group Lie algebra, $\mathrm{C}^{e b n} u_{e} v_{b}=0$. First, we rearrange Eq. (3.5) into the form

$$
\mathrm{B}_{[b c}{ }^{n} \mathrm{~B}_{d] n a}=2 \mathrm{C}_{n a}^{p} \mathrm{C}_{[c}^{n e} V_{b|e|} V_{d] p}+2 \mathrm{C}_{a[d}{ }^{p} \mathrm{C}_{c}{ }^{n e} V_{b] e} V_{n p}
$$

Substituting $V_{e b}=2 u_{[b} v_{e]}$ leads to

$$
\mathrm{B}_{[b c}^{n} \mathrm{~B}_{d] n a}=4 \mathrm{C}_{n a}^{p} \mathrm{C}_{[c}^{n e} u_{b} v_{d]} u_{[e} v_{p]}+2 \mathrm{C}_{a[d}^{p} \mathrm{C}_{c}^{n e} u_{b]} u_{n} v_{e} v_{p}-2 \mathrm{C}_{a[d}{ }^{p} \mathrm{C}_{c}^{n e} v_{b]} u_{n} v_{e} u_{p}
$$

The last two terms directly vanish since $\mathrm{C}_{c}{ }^{n e} u_{n} v_{e}=0$. The first term can be rearranged by the Jacobi property $2 \mathrm{C}_{c}{ }^{n[e} \mathrm{C}_{n a}{ }^{p]}=\mathrm{C}_{n}{ }^{p e} \mathrm{C}_{a c}{ }^{n}$, yielding $2 \mathrm{C}_{n}{ }^{e p} \mathrm{C}_{a[c}{ }^{n} u_{b} v_{d]} u_{e} v_{p}$ which vanishes 
since $\mathrm{C}_{n}{ }^{e p} u_{e} v_{p}=0$. This demonstrates the Jacobi property $0=\mathrm{B}_{[b c}{ }^{n} \mathrm{~B}_{d] n a}$. More generally, the same property can be shown to hold if $V_{e b}$ has the form $2 u_{[e} v_{b]}+2 x_{[e} y_{b]}+\cdots$ such that $u_{e}, v_{b}, x_{e}, y_{b}, \ldots$ all commute in the gauge group Lie algebra.

Finally, for $V_{e b}=2 u_{[e} v_{b]}$, we show that if the commutator of $u_{e}$ and $v_{b}$ in the gauge group Lie algebra is nonvanishing, $\mathrm{C}^{e b n} u_{e} v_{b} \neq 0$, the Jacobi property $(3.5)$ on $\mathrm{B}_{b c d}=2 V_{e[b} \mathrm{C}_{c] d} e$ implies $u_{e}$ and $v_{b}$ belong to an invariant $S U(2)$ subalgebra. Hence, in satisfying the Jacobi property, a vanishing commutator of $u_{e}$ and $v_{b}$ is a necessary as well as sufficient requirement in any simple gauge group Lie algebra other than $S U(2)$.

To proceed, we suppose $x^{n}=\mathrm{C}^{e b n} u_{e} v_{b}$ is nonvanishing and $k^{e b} u_{e} v_{b}=0$. The Jacobi property (3.5) then leads to

$$
0=x^{e} \mathrm{C}_{e[c}{ }^{d} u_{b} v_{a]}+\mathrm{C}_{[a}^{p d} v_{b} x_{c]} u_{p}+\mathrm{C}_{[a}^{p d} u_{b} x_{c]} v_{p}
$$

First we contract this expression by $v^{a} u^{b} y^{c}$ with an arbitrary $y^{c}$ satisfying $u_{a} y^{a}=0=v_{a} y^{a}$. This yields $0=x^{e} y^{c} \mathrm{C}_{e c}{ }^{d}$, which implies $x^{e} \mathrm{C}_{e c d}$ is proportional to $u_{[c} v_{d]}$. As a result, we have

$$
x^{e} \mathrm{C}_{e c d}=2 u_{[c} v_{d]}
$$

The expression (A8) now simplifies to $0=x_{[c}\left(\mathrm{C}_{a}{ }^{p d} v_{b]} u_{p}+\mathrm{C}_{a}^{p d} u_{b]} v_{p}\right)$. Contracting by $x^{c} u^{b}$ and then using the relation (A9) leads to

$$
u^{2} v_{p} \mathrm{C}_{a d}^{p}=2 x_{[a} u_{d]}
$$

where $u^{2}=k^{e b} u_{e} u_{b}$. Similarly,

$$
v^{2} u_{p} \mathrm{C}_{a d}^{p}=-2 x_{[a} v_{d]}
$$

Taken together, the relations (A9), (A10), (A11) imply $\left\{u^{a}, v^{b}, x^{c}\right\}$ span an invariant $S U(2)$ subalgebra in the gauge group Lie algebra.

\section{APPENDIX B: STRUCTURE OF THE AUXILIARY LIE ALGEBRA}

The form (3.7) for the structure constants $\mathrm{B}_{b c}{ }^{a}$ in the general case of a semi-simple gauge group Lie algebra yields an auxiliary Lie algebra multiplication which differs in several 
features compared to the $S U(2)$ case. In terms of a Lie algebra basis $\mathbf{e}_{a}$ associated to the structure constants $\mathrm{C}_{b c}{ }^{a}$, with multiplication $\left[\mathbf{e}_{b}, \mathbf{e}_{c}\right]=\mathrm{C}_{b c}{ }^{a} \mathbf{e}_{a}$ and Killing metric $\left(\mathbf{e}_{b}, \mathbf{e}_{c}\right)=$ $k_{b c}$, the auxiliary multiplication is given by

$$
\left[\mathbf{e}_{b}, \mathbf{e}_{c}\right]_{B}=2\left(\mathbf{u}, \mathbf{e}_{[b}\right)\left[\mathbf{e}_{c]}, \mathbf{v}\right]-2\left(\mathbf{v}, \mathbf{e}_{[b}\right)\left[\mathbf{e}_{c]}, \mathbf{u}\right]
$$

where $\mathbf{u}=\delta^{b a} u_{b} \mathbf{e}_{a}$ and $\mathbf{v}=\delta^{b a} v_{b} \mathbf{e}_{a}$ are Lie algebra vectors associated to $u_{b}$ and $v_{b}$, which satisfy $[\mathbf{u}, \mathbf{v}]=0$. We can understand the structure of this multiplication in terms of $\mathbf{u}$ and $\mathbf{v}$ as follows.

First, we note the commutator expression (B1) depends on $\mathbf{u}$ and $\mathbf{v}$ antisymmetrically, so it remains invariant under $\mathbf{u} \rightarrow a \mathbf{u}+b \mathbf{v}, \mathbf{v} \rightarrow c \mathbf{v}+d \mathbf{u}$ such that $a c-b d=1$. Using this invariance we set $(\mathbf{u}, \mathbf{v})=0$ for convenience. Next, we let $\mathbf{H}$ define the subspace in the gauge group Lie algebra such that

$$
\begin{aligned}
& (\mathbf{u}, \mathbf{H})=0=(\mathbf{v}, \mathbf{H}) \\
& {[\mathbf{u}, \mathbf{H}]=0=[\mathbf{v}, \mathbf{H}]}
\end{aligned}
$$

Thus, vectors in $\mathbf{H}$ are orthogonal to and commute with $\mathbf{u}$ and $\mathbf{v}$. We then let $\mathbf{H}_{\perp}$ define the subspace orthogonal to $\mathbf{u}, \mathbf{v}, \mathbf{H}$ in the gauge group Lie algebra,

$$
\begin{aligned}
& \left(\mathbf{u}, \mathbf{H}_{\perp}\right)=0=\left(\mathbf{v}, \mathbf{H}_{\perp}\right) \\
& \left(\mathbf{H}, \mathbf{H}_{\perp}\right)=0
\end{aligned}
$$

The spaces $\mathbf{H}, \mathbf{H}_{\perp}$, and vectors $\mathbf{u}, \mathbf{v}$ together span the gauge group Lie algebra. The property that the gauge group Lie algebra is semi-simple implies

$$
\left[\mathbf{u}, \mathbf{H}_{\perp}\right] \subseteq \mathbf{H}_{\perp},\left[\mathbf{v}, \mathbf{H}_{\perp}\right] \subseteq \mathbf{H}_{\perp}
$$

Now, from the basis multiplication (B1), we have the following auxiliary Lie algebra multiplication. For vectors $\mathbf{h}, \mathbf{g}$ in $\mathbf{H}$ and vectors $\mathbf{x}, \mathbf{y}$ in $\mathbf{H}_{\perp}$,

$$
[\mathbf{u}, \mathbf{h}]_{B}=0,[\mathbf{v}, \mathbf{h}]_{B}=0,[\mathbf{h}, \mathbf{g}]_{B}=0
$$


using the orthogonality ( $\mathrm{B} 2)$ and commutativity $(\mathbb{B} 3)$, while

$$
\begin{aligned}
& {[\mathbf{u}, \mathbf{x}]_{B}=-|\mathbf{u}|^{2}[\mathbf{v}, \mathbf{x}],[\mathbf{v}, \mathbf{x}]_{B}=|\mathbf{v}|^{2}[\mathbf{u}, \mathbf{x}]} \\
& {[\mathbf{x}, \mathbf{y}]_{B}=0,[\mathbf{x}, \mathbf{h}]_{B}=0}
\end{aligned}
$$

using the orthogonality $(\bar{B} 4)$ and $(B 5)$, where $|\mathbf{u}|^{2}=(\mathbf{u}, \mathbf{u})$ and $|\mathbf{v}|^{2}=(\mathbf{v}, \mathbf{v})$. In addition,

$$
[\mathbf{u}, \mathbf{v}]_{B}=0
$$

Algebraically, these commutators show that $\mathbf{u}, \mathbf{v}$ together define an abelian Lie subalgebra A, while $\mathbf{H}$ and $\mathbf{H}_{\perp}$ also define abelian Lie subalgebras, such that $\mathbf{A}$ commutes with $\mathbf{H}$ and acts invariantly on $\mathbf{H}_{\perp}$, and the subalgebras $\mathbf{H}, \mathbf{H}_{\perp}$ commute. Hence the span of $\mathbf{A}, \mathbf{H}, \mathbf{H}_{\perp}$ defines an auxiliary Lie algebra which is the product of $\mathbf{H}$ with the semi-direct product of $\mathbf{A}$ and $\mathbf{H}_{\perp}$ where $\frac{1}{|\mathbf{u}|^{2}} \mathbf{u}$ acts on $\mathbf{H}_{\perp}$ by multiplication by $-\mathbf{v}$ while $\frac{1}{|\mathbf{v}|^{2}} \mathbf{v}$ acts on $\mathbf{H}_{\perp}$ by multiplication by $\mathbf{u}$. 


\section{REFERENCES}

* email: anco@math.ubc.ca

[1] S.C. Anco, J. Math. Phys. 36 (1995) 6553-6565.

[2] D.Z. Freedman and P.K. Townsend, Nuc. Phys. B 177 (1981) 282-296.

[3] As additional structure, an $S U(2)$ bundle over the manifold is needed. This bundle is assumed to be globally trivial for simplicity.

[4] Curiously, this auxiliary Lie alebra is isomorphic to the Lie algebra of translations and dilations in Euclidean $R^{3}$ space, with $\mathbf{w}_{1}$ and $\mathbf{w}_{2}$ identified as the generators of translations and $\frac{1}{|\mathbf{v}|^{2}} \mathbf{v}$ identified as the generator of dilations.

[5] Y. Choquet-Bruhat, C. DeWitt-Morrette, Analysis, manifolds, and physics (NorthHolland, Amerstdam).

[6] A Lie algebra is simple if and only if it has no proper invariant subalgebras.

[7] S.C. Anco, Contemp. Math. 132 (1992) 27-50.

[8] In preparation. 